\title{
COMPARACIÓN DE SISTEMAS DE PUNTAJE PRONÓSTICO EN LA PREDICCIÓN DE MORTALIDAD Y COMPLICACIONES EN SEPSIS
}

\author{
Diego Marin-Marín',a, Alonso Soto ${ }^{1,2, b}$
}

\begin{abstract}
RESUMEN
Objetivos. Comparar los sistemas de puntaje MEDS (Mortality in Emergency Department Sepsis), SOFA (Sepsis-related Organ Failure Assessment), APACHE II (Acute Physiology and Chronic Health Evaluation) y CURB-65 (Confusion, Urea, Respiratory rate, Blood pressure, 65 years of age and older) para pronóstico de mortalidad y complicaciones en pacientes sépticos. Materiales y métodos. Se realizó un estudio prospectivo de evaluación de prueba diagnóstica. Se realizó la comparación de los puntajes mediante el análisis de las curvas ROC (receiver operating characteristic) correspondientes a cada uno de ellos, teniendo como variable dependiente el desarrollo de muerte o complicaciones. Resultados. 265 pacientes fueron incluidos en el análisis; 155 (58,4\%) pacientes fallecieron y/o presentaron complicaciones. En el análisis multivariado se encontró como variables asociadas a mortalidad y complicaciones a la edad, oliguria, alteración del estado mental, daño pulmonar agudo y el uso de inotrópicos. Con respecto al análisis de las curvas ROC, el área bajo la curva ROC fue 0,74 (IC 95\%: 0,68-0,80) para SOFA, 0,73 (IC 95\%: 0,67-0,79) para MEDS, 0,73 (IC 95\%: 0,660,79) para APACHE II, y 0,67 (IC 95\%: 0,60-0,73) para CURB-65 siendo este último significativamente inferior a las tres primeras. Conclusiones. Los puntajes MEDS, SOFA y APACHE II son puntajes adecuados para predecir mortalidad y complicaciones en pacientes sépticos y deben considerarse su uso en la atención de emergencias en hospitales de Perú.
\end{abstract}

Palabras clave: Sepsis; Predicción; /diagnóstico; Pronóstico; /mortalidad (fuente: DeCS BIREME).

\section{COMPARISON OF PROGNOSTIC SCORING SYSTEMS IN THE PREDICTION OF MORTALITY AND COMPLICATIONS FROM SEPSIS}

\begin{abstract}
Objectives. To compare the Mortality in Emergency Department Sepsis (MEDS), Sepsis-related Organ Failure Assessment (SOFA), Acute Physiology and Chronic Health Evaluation (APACHE II), and Confusion, Urea, Respiratory rate, Blood pressure, 65 years of age and older (CURB-65) scoring systems for predicting mortality and complications in septic patients. Materials and methods. A prospective study of diagnostic test assessment was carried out. Scores were compared by analysis of receiver operating characteristic (ROC) curves corresponding to each score, with the development of death or complications as the dependent variable. Results. There were 265 patients included in the analysis; $155(58.4 \%)$ patients died and/or presented complications. The multivariate analysis revealed variables associated with mortality and complications were age, oliguria, mental health impairment, acute lung injury, and the use of inotropes. Analysis of ROC curves showed the area below the ROC curve was 0.74 (95\% Cl: $0.68-0.80)$ for SOFA, 0.73 (95\% Cl: 0.67-0.79) for MEDS, 0.73 (95\% Cl: 0.66-0.79) for APACHE II, and 0.67 (95\% Cl: 0.60-0.73) for CURB-65, the latter being significantly lower than the first three. Conclusions. The MEDS, SOFA, and APACHE II are adequate scoring systems for predicting mortality and complications in septic patients. It is essential to utilize these scoring systems in emergency medical assistance in hospitals in Peru.
\end{abstract}

Key words: Sepsis; Forecasting; /diagnosis; Prognosis; /mortality (source: MESH NLM).

\section{INTRODUCCIÓN}

La sepsis sigue siendo una de las principales causas de muerte en pacientes en estado crítico en los servicios de emergencia ${ }^{(1)}$, entre el 20 a $50 \%$ de los pacientes gravemente afectados fallecen, además de reducir la calidad de vida de los que sobreviven (2). La sepsis tiene una prevalencia muy alta, tanto en los pacientes

\footnotetext{
Facultad de Medicina Humana, Universidad Ricardo Palma. Lima, Perú.

Hospital Nacional Hipólito Unanue. Lima, Perú.

Médico cirujano, ${ }^{\mathrm{b}}$ médico internista, $\mathrm{PhD}$ en Ciencias Médicas.

Este estudio ha sido presentado como tesis para obtener el grado de médico cirujano en la Universidad Ricardo Palma por el autor principal. El resumen ha sido presentado en el XXIV Curso Internacional del Policlínico Peruano Japonés y V Curso Internacional de la Clínica Centenario Peruano Japonesa "De la evidencia a la práctica en medicina", realizado en abril de 2014.

Recibido: 14-07-15 Aprobado: 04-11-15
}

Citar como: Marin-Marín D, Soto A. Comparación de sistemas de puntaje pronóstico en la predicción de mortalidad y complicaciones en sepsis. Rev Peru Med Exp Salud Publica. 2016;33(1):51-7. doi: 10.17843/rpmesp.2016.331.1924 
hospitalizados como en los pacientes de la unidad de cuidados intensivos $(\mathrm{UCl})^{(3-5)}$. Más de la mitad de todos los pacientes sépticos desarrollan sepsis grave y un cuarto desarrolla shock séptico. La incidencia global de la sepsis es de aproximadamente 300 casos $/ 10^{5}$ habitantes/año en los Estados Unidos. En España se han reportado 367 casos $/ 10^{5}$ habitantes/año ${ }^{(4)}$. En Chile se encontró una prevalencia de $32,5 \%$ de pacientes con sepsis grave en la $\mathrm{UCl}^{(6)}$.

La atención a pacientes sépticos representa una gran carga económica para el estado y el sistema de salud en general (4). Asimismo, representa un reto para los proveedores y administradores del cuidado de la salud ${ }^{(5)}$.

Las reglas de predicción clínica (RPC) son herramientas diseñadas para la toma de decisiones, estas contienen tres o más variables simples obtenidas a partir de la historia clínica, examen físico y/o exámenes auxiliares. Dichas reglas son creadas, usualmente, por análisis multivariado, pueden predecir la mortalidad de una enfermedad y sugerir un diagnóstico o curso terapéutico de acción ${ }^{(7)}$. La mayor parte de RPC corresponde a sistemas de puntaje (scores). Entre las principales RPC propuestas para la evaluación del pronóstico en pacientes con sepsis se encuentran los sistemas de puntaje MEDS (Mortality in Emergency Department Sepsis) ${ }^{(8)}$, SOFA (Sepsis-related Organ Failure Assessment) (9), APACHE II (Acute Physiology and Chronic Health Evaluation) (10) y CURB-65 (Confusion, Urea, nitrogen, Respiratory rate, Blood pressure, 65 years of age and older) ${ }^{(11)}$.

Al no disponer de un marcador pronóstico único, es importante disponer de herramientas clínicas para estimar la probabilidad de muerte intrahospitalaria en pacientes sépticos y así identificar los pacientes de alto riesgo y mejorar el uso apropiado de las intervenciones. Entre estas herramientas, las RPC son comúnmente utilizadas tanto en el servicio de emergencia como en la UCl ${ }^{(1,12-15)}$. El puntaje MEDS ha sido desarrollado específicamente para predecir la mortalidad a 28 días en pacientes del servicio de emergencia con sospecha de infección $(8,16)$ e incluye como variables la presencia de enfermedad terminal, edad, recuento de abastonados, taquipnea o hipoxia, shock, conteo plaquetario, alteración del estado mental, residencia de hogar de ancianos e infección de las vías respiratorias inferiores ${ }^{(1,8,12,13,17)}$.

El puntaje APACHE II, fue descrito por Knaus y colaboradores en $1985^{(10,18)}$. Se basa en doce mediciones fisiológicas de rutina, junto con la edad y estado de salud previo dentro de las primeras $24 \mathrm{~h}$ a la admisión del paciente ${ }^{(18)}$.

El puntaje SOFA fue creado por el Grupo de Trabajo sobre Problemas Relacionados con la Sepsis de la Sociedad Europea de Medicina Intensiva en $1996^{(9,19)}$. El puntaje evalúa seis sistemas orgánicos dependiendo del nivel de disfunción: respiratorio, circulatorio, renal, hematológico, hepático y neurológico ${ }^{(19,20)}$.

El puntaje CURB-65, que se desarrolló en el año 2003 para el diagnóstico de pacientes con neumonía, incorpora como variables la confusión, urea, frecuencia respiratoria, presión arterial y edad ${ }^{(1,11)}$. El uso del puntaje CURB-65 como predictor de mortalidad ha sido evaluado en pacientes con neumonía y en pacientes con cualquier sospecha de infección ${ }^{(1)}$.

Todos estos puntajes tienen estudios disímiles con respecto a su capacidad de predicción en sepsis, la cual puede ser desde muy pobre hasta excelente ${ }^{(1,4,8,12-15,17,19-23)}$.

El objetivo del estudio fue comparar los sistemas de puntaje MEDS, SOFA, APACHE II y CURB-65 para pronóstico de mortalidad y complicaciones en pacientes con diagnóstico de sepsis admitidos en el servicio de emergencia de un hospital peruano.

\section{MATERIALES Y MÉTODOS}

\section{DISEÑO DE ESTUDIO}

Se realizó un estudio prospectivo de evaluación de prueba diagnóstica.

\section{POBLACIÓN Y MUESTRA}

La población estuvo constituida por los pacientes con diagnóstico de sepsis atendidos en el área de emergencia del Hospital Nacional Hipólito Unanue (HNHU), un centro de referencia en Lima, Perú. El cálculo del tamaño muestral se realizó en base a una diferencia estimada del $10 \%$ entre la exactitud de los puntajes, un error tipo a de: 0,05 y una potencia del $80 \%$. De acuerdo a ello, el tamaño muestral requerido fue de 199 pacientes. Sin embargo, a fin de incrementar la potencia estadística del estudio, facilitar el estudio de subgrupos y considerando las posibles pérdidas en el seguimiento y posibles datos incompletos se amplió el tamaño muestral a 250 pacientes.

\section{CRITERIOS DE INCLUSIÓN Y EXCLUSIÓN}

Para el diagnóstico de síndrome de respuesta inflamatoria sistémica (SRIS), sepsis, sepsis grave y shock séptico se usaron los criterios de acuerdo al consenso de $1992^{(3)}$. Se incluyeron a pacientes mayores de 18 años con un tiempo esperado de permanencia en emergencia mayor a $6 \mathrm{~h}$ que cumplieron con dos o más criterios de síndrome de respuesta inflamatoria sistémica más evidencia de foco infeccioso, y que aceptaron el consentimiento informado. Aquellos pacientes con incapacidad para aceptar el consentimiento informado fueron aceptados por su familiar. Se excluyeron a pacientes con parámetros incompletos para cuantificar 
los puntajes, sedoanalgesia al momento de la primera evaluación y evidencia de lesión traumática.

\section{VARIABLES}

La variable dependiente utilizada fue la combinación de mortalidad o complicaciones (incluyendo ingreso a la unidad de cuidados intensivos, requerimiento de ventilación mecánica e ingreso a hemodiálisis). Entre las variables independientes se utilizaron variables demográficas, clínicas y de laboratorio, así como los resultados de los puntajes SOFA, APACHE II, MEDS y CURB-65. El cálculo de los puntajes en cada paciente se realizó en las primeras $24 \mathrm{~h}$ de ingresados a la emergencia.

\section{PROCEDIMIENTOS}

El método para el control de la calidad de datos fue la elaboración de una base de datos en Microsoft Access; utilizando validación a través de la programación de criterios específicos de llenado completo de datos, rangos preespecificados y coherencia interna.

\section{ANÁLISIS ESTADÍSTICO}

El análisis univariado se realizó con medidas de tendencia central (media) y dispersión (desviación estándar) de acuerdo a la distribución de los datos. En el análisis bivariado se evaluó la asociación entre las variables dependientes e independientes mediante las pruebas de chi cuadrado y la t de Student para variables categóricas y numéricas respectivamente. El análisis multivariado se realizó mediante el uso de modelos de regresión logística múltiple. La evaluación de los puntajes se realizó mediante el análisis y comparación de áreas bajo la curva ROC. Se consideró un valor de $p<0,05$ como significativo y $p<0,01$ como altamente significativo. Para el análisis estadístico se utilizó el programa STATA versión 11 (Stata Corporation, College Station, TX).

\section{CONSIDERACIONES ÉTICAS}

El estudio contempló las recomendaciones de la declaración de Helsinki. Asimismo, el Comité Institucional de Ética en Investigación del HNHU aprobó tanto el estudio, como el formulario de consentimiento informado, el cual fue obtenido de todos los participantes (o familiares de primer grado en caso de pacientes con trastorno de sensorio).

\section{RESULTADOS}

Se incluyeron 265 pacientes con una media de edad de 63 años y una desviación estándar de 20 , correspondiendo $136(51,3 \%)$ al sexo masculino. Las características demográficas y clínicas son presentadas en las tablas 1 a 3. En nuestro estudio, 119 (45\%) pacientes fallecieron y

Tabla 1. Comparación de características demográficas, foco infeccioso y variables clínicas entre los pacientes con diagnóstico de sepsis de acuerdo a la presencia o no de mortalidad y/o complicaciones

\begin{tabular}{|c|c|c|c|c|}
\hline & Total $(n=265)$ & $\begin{array}{c}\text { Mortalidad y/o } \\
\text { complicaciones presentes } \\
(n=155)\end{array}$ & $\begin{array}{c}\text { Mortalidad y/o } \\
\text { complicaciones } \\
\text { ausentes }(n=110)\end{array}$ & Valor $p$ \\
\hline \multicolumn{5}{|l|}{$\begin{array}{l}\text { Características } \\
\text { demográficas }\end{array}$} \\
\hline Edad media (de) & $62,9(19,9)$ & $66,7(19,2)$ & $57,7(19,9)$ & $<0,001$ \\
\hline Procedente de casa de reposo (\%) & $1(0,3)$ & $0(0,0)$ & $1(0,9)$ & 0,41 \\
\hline $\begin{array}{l}\text { Media de estancia hospitalaria en } \\
\text { días (de) }\end{array}$ & $17,4(16,8)$ & $16,2(17,9)$ & $19,1(15,0)$ & 0,16 \\
\hline \multicolumn{5}{|l|}{ Foco infeccioso, $n(\%)$} \\
\hline Respiratorio & $202(76,2)$ & $130(83,8)$ & $72(65,4)$ & 0,001 \\
\hline Urinario & $59(22,2)$ & $36(23,2)$ & $23(20,9)$ & 0,65 \\
\hline Dérmico & $38(14,3)$ & $20(12,9)$ & $18(16,3)$ & 0,42 \\
\hline Abdominal & $32(12)$ & $17(10,9)$ & $15(13,6)$ & 0,51 \\
\hline Meníngeo & $4(1,5)$ & $2(1,2)$ & $2(1,8)$ & 1,00 \\
\hline Catéter venoso central & $4(1,5)$ & $3(1,9)$ & $1(0,9)$ & 0,64 \\
\hline \multicolumn{5}{|l|}{ Variables clínicas, n (\%) } \\
\hline Injuria pulmonar aguda & $146(55,0)$ & $102(65,8)$ & $44(40,0)$ & $<0,001$ \\
\hline Uso de inotrópicos & $60(22,6)$ & $48(30,9)$ & $12(10,9)$ & $<0,001$ \\
\hline Alteración del estado mental & $117(44,1)$ & $84(54,1)$ & $33(30,0)$ & $<0,001$ \\
\hline Oliguria & $33(13,1)$ & $26(17,9)$ & $7(6,6)$ & $<0,01$ \\
\hline Lactato $(\mathrm{mmol} / \mathrm{l})$, media $(\mathrm{de})$ & $3,2(2,4)$ & $3,3(2,4)$ & $2,9(2,3)$ & 0,15 \\
\hline
\end{tabular}

de: desviación estándar 
Tabla 2. Frecuencia de comorbilidades entre los pacientes con diagnóstico de sepsis de acuerdo a la presencia o no de mortalidad y/o complicaciones

\begin{tabular}{|c|c|c|c|c|}
\hline Comorbilidades, n (\%) & Total $(n=265)$ & $\begin{array}{c}\text { Mortalidad y/o } \\
\text { complicaciones } \\
\text { presentes }(n=155)\end{array}$ & $\begin{array}{c}\text { Mortalidad y/o } \\
\text { complicaciones } \\
\text { ausentes }(n=110)\end{array}$ & Valor $p$ \\
\hline Diabetes mellitus tipo 2 & $54(20,3)$ & $30(19,3)$ & $24(21,8)$ & 0,62 \\
\hline Hipertensión arterial & $79(29,8)$ & $54(34,8)$ & $25(22,7)$ & 0,03 \\
\hline Insuficiencia renal crónica & $31(11,7)$ & $27(17,4)$ & $4(3,6)$ & $<0,001$ \\
\hline $\begin{array}{l}\text { Enfermedad cerebrovascular } \\
\text { isquémica }\end{array}$ & $23(8,6)$ & $15(9,6)$ & $8(7,2)$ & 0,49 \\
\hline Insuficiencia cardiaca & $26(9,8)$ & $16(10,3)$ & $10(9,0)$ & 0,74 \\
\hline $\begin{array}{l}\text { Enfermedad pulmonar obstructiva } \\
\text { crónica }\end{array}$ & $4(1,5)$ & $3(1,9)$ & $1(0,9)$ & 0,64 \\
\hline $\begin{array}{l}\text { Enfermedad pulmonar intersticial } \\
\text { difusa }\end{array}$ & $19(7,1)$ & $11(7,1)$ & $8(7,2)$ & 0,96 \\
\hline Cirrosis hepática & $10(3,7)$ & $7(4,5)$ & $3(2,7)$ & 0,53 \\
\hline
\end{tabular}

Tabla 3. Frecuencia de disfunción orgánica entre los pacientes con diagnóstico de sepsis de acuerdo a la presencia o no de mortalidad y/o complicaciones

\begin{tabular}{lcccc}
\hline $\begin{array}{c}\text { Tipos de falla orgánica } \\
\text { presente, } \mathbf{n}(\%)\end{array}$ & Total $(\mathbf{n = 2 6 5 )}$ & $\begin{array}{c}\text { Mortalidad y/o } \\
\text { complicaciones } \\
\text { presentes }(\mathbf{n = 1 5 5 )}\end{array}$ & $\begin{array}{c}\text { Mortalidad y/o } \\
\text { complicaciones } \\
\text { ausentes }(\mathbf{n = 1 1 0})\end{array}$ & Valor $\boldsymbol{p}$ \\
\hline Neurológica & $117(44,1)$ & $84(54,1)$ & $33(30,0)$ & $<0,001$ \\
\hline Renal & $77(29,1)$ & $57(37,0)$ & $20(18,1)$ & 0,001 \\
Hematológica & $67(25,2)$ & $52(33,5)$ & $15(13,6)$ & $<0,001$ \\
\hline Respiratoria & $61(23,0)$ & $60(38,7)$ & $1(0,9)$ & $<0,001$ \\
\hline Cardiovascular & $59(22,2)$ & $48(30,9)$ & $11(10,0)$ & $<0,001$ \\
\hline Hepática & $27(10,3)$ & $21(13,8)$ & $6(5,4)$ & 0,03 \\
Metabólica & $26(9,9)$ & $21(13,7)$ & $5(4,5)$ & 0,02 \\
\hline
\end{tabular}

$155(58,4 \%)$ fallecieron y/o presentaron complicaciones. En cuanto a las complicaciones, 11 (4\%) pacientes ingresaron a la $\mathrm{UCl}$; 60 (23\%) requirieron ventilación mecánica y 15 (6\%) ingresaron a hemodiálisis. El 33,9\% de pacientes presentó sepsis sin criterios de gravedad, $44,5 \%$ sepsis grave y $21,5 \%$ shock séptico. El foco infeccioso más frecuente fue el respiratorio.

La media de los puntajes MEDS, SOFA, APACHE II y CURB-65 fue 8, 5, 16 y 2, respectivamente, siendo en todos los casos significativamente mayores en aquellos pacientes con mortalidad o complicaciones (Tabla 4). En el análisis multivariado se encontró como predictores significativos de mortalidad y complicaciones a la edad $(p=0,04)$, oliguria $(p=0,04)$, alteración del estado mental $(p=0,01)$, daño pulmonar agudo $(p=0,02)$ y el uso de inotrópicos $(p=0,005)$.

Con respecto al análisis de las curvas $\mathrm{ROC}$, el área bajo la curva ROC fue 0,74 para SOFA, 0,73 para MEDS, 0,73 para APACHE II y 0,67 para CURB-65 (Figura 2). No se encontraron diferencias significativas entre MEDS, SOFA y APACHE II $(p=0,88)$, sin embargo, las áreas bajo la curva ROC fueron significativamente mayores en los puntajes MEDS, SOFA y APACHE II con respecto al CURB-65.

Con respecto a la evaluación de los puntos de corte óptimos para la predicción de mortalidad o complicaciones, para el score MEDS un valor $\leq 5$ puntos se asoció a una menor probabilidad de muerte y complicaciones (LRde 0,08$)$ mientras que un score $\geq 13$ puntos aumentó significativamente dicho riesgo ( $L R+$ de 5,1). En relación al score SOFA un valor $\leq 1$ punto se asoció a una menor probabilidad de muerte y complicaciones (LR- de 0,12) mientras que un score $\geq 13$ aumentó significativamente dicho riesgo (LR+ de 5,06). Con respecto al score APACHE II un valor $\leq 10$ puntos se asoció a una menor probabilidad de muerte y complicaciones (LR- de 0,09).

\section{DISCUSIÓN}

El principal hallazgo de nuestro estudio es que al evaluar los puntajes pronósticos, los puntajes MEDS, SOFA y APACHE II son similares y adecuados para predecir mortalidad y complicaciones en pacientes 
Tabla 4. Comparación de puntajes entre los pacientes con diagnóstico de sepsis de acuerdo a la presencia o no de mortalidad y/o complicaciones

\begin{tabular}{lcccc}
\hline \multicolumn{1}{c}{ Puntajes, media (de) } & Total $(\mathbf{n = 2 6 5 )}$ & $\begin{array}{c}\text { Mortalidad y/o } \\
\text { complicaciones } \\
\text { presentes (n=155) }\end{array}$ & $\begin{array}{c}\text { Mortalidad y/o } \\
\text { complicaciones } \\
\text { ausentes (n=110) }\end{array}$ & Valor $\boldsymbol{p}$ \\
\hline MEDS & $8,2(3,6)$ & $9,5(3,3)$ & $6,5(3,2)$ & $<0,001$ \\
SOFA & $5,0(3,5)$ & $6,2(3,4)$ & $3,4(3,1)$ & $<0,001$ \\
APACHE II & $16,2(7,3)$ & $18,6(6,6)$ & $12,8(6,9)$ & $<0,001$ \\
CURB-65 & $2,3(1,1)$ & $2,6(1,0)$ & $2,0(1,0)$ & $<0,001$ \\
\hline
\end{tabular}

MEDS: Mortality in Emergency Department Sepsis

SOFA: Sepsis-related Organ Failure Assessment

CURB-65: Confusion, Urea, Respiratory rate, Blood pressure, 65 years of age and older

de: desviación estándar

con diagnóstico de sepsis. A pesar de ser un score interesante, porque utiliza pocas variables para su puntuación, el score CURB-65 presenta una capacidad diagnóstica significativamente inferior a los primeros. Siendo los puntajes MEDS, SOFA y APACHE II similares en cuanto a rendimiento diagnóstico; el score más fácil de usar es posiblemente el score MEDS, ya que sus variables están fácilmente disponibles en el momento de la evaluación en el servicio de emergencia ${ }^{\left({ }^{8}\right)}$. Por ello, sugerimos que se use como una herramienta de predicción clínica en la emergencia. El uso de la variable casa de reposo podría no estar acorde a la realidad peruana por lo que en nuestro medio podría utilizarse una modificación del score que no incluya la procedencia de casa de reposo. Sin embargo, ello debería ser validado prospectivamente.

Llama la atención la alta mortalidad en pacientes con sepsis encontrada en nuestro estudio en comparación con otras publicaciones ${ }^{(1,13,15,24)}$. Una explicación podría ser la enorme carencia de camas en la UCI. De hecho, solo $11(4 \%)$ de pacientes sépticos incluidos en nuestro estudio tuvo acceso a dicha unidad, pese a haberse presentado 56 casos de shock séptico y 60 pacientes en ventilación mecánica los cuales fueron en su gran mayoría tratados en el servicio de emergencia y pisos de hospitalización. Otro factor involucrado podría ser la falta de un manejo estandarizado basado en guías nacionales sustentadas en la mejor evidencia disponible. Nuestro estudio no contó con la potencia suficiente para un análisis específico de mortalidad, por lo que es necesario estudios multicéntricos con mayor número de pacientes a fin de clarificar los factores asociados a esta. Nuestros hallazgos incluyeron a la edad avanzada, oliguria, alteración del estado mental, daño pulmonar agudo y uso de inotrópicos como predictores independientes más importantes de muerte o complicaciones.

Otro aspecto que llama la atención es la gran proporción de pacientes con sepsis grave y shock séptico en comparación con otras series ${ }^{(1,15)}$. Ello se puede explicar por el tipo de población que acude al HNHU, el cual es el único centro de referencia para una zona con más de 2 millones de habitantes, que atiende una gran cantidad de pacientes referidos de centros de menor nivel, y donde se atienden la mayoría de casos de sepsis no graves.

Existen otros estudios que han comparado los sistemas de puntaje evaluados en nuestro estudio. Entre aquellos que han comparado MEDS con APACHE II se han encontrado hallazgos disímiles. Mientras que algunos han encontrado que APACHE II tiene un mejor rendimiento diagnóstico que el MEDS ${ }^{(15,25)}$ otros han evidenciado lo contrario ${ }^{(13)}$. Otros estudios compararon MEDS con CURB-65 (1,12) encontrando un mejor rendimiento del puntaje MEDS concordando con nuestros hallazgos. Un estudio más reciente corrobora la utilidad de ambos sistemas de puntaje para la predicción de mortalidad a 28 días en pacientes atendidos en emergencia ${ }^{(30)}$. Otro estudio ha comparado el puntaje APACHE II con SOFA ${ }^{(20)}$ encontrando un mejor rendimiento del primero mientras que una publicación más reciente encontró que el SOFA es mejor que los puntajes MEDS y CURB-65 (28). Las discordancias en la performance de los diferentes sistemas de puntaje pueden explicarse por diferencias en las poblaciones o escenarios clínicos en los que son evaluados y enfatiza la necesidad de evaluar y validar estas herramientas localmente antes de recomendar su uso generalizado.

Existen otros sistemas de puntaje que se han desarrollado para sepsis en emergencia tales como el MPM II ${ }_{0}$ (Mortality Prediction Model), SAPS II (Simplified Acute Physiology Score), REMS (Rapid Emergency Medicine Score), PIRO (Predisposition, Insult/Infection, Response, and Organ dysfunction) ${ }^{(1,15,26)}$, sin embargo, son pocos conocidos o aplicados en el medio peruano. Nuestro estudio fue diseñado para evaluar reglas de predicción clínica de uso habitual en el ámbito hospitalario nacional, pero que no suelen utilizarse para la evaluación pronóstica rutinaria específicamente en sepsis. La implementación de RPC no es sencilla, pero 
puede optimizarse al utilizar herramientas ya conocidas por el clínico. La intervención temprana en la sepsis es muy importante para la supervivencia del paciente, sin embargo, la demora continúa siendo frecuente ${ }^{(26)}$. Las RPC son herramientas valiosas que puede permitir intervenir tempranamente, particularmente en aquellos pacientes identificados como de alto riesgo para mortalidad y complicaciones. Existen otras medidas que pueden contribuir a mejorar el manejo de pacientes con sepsis en adición a las RPC. Por ejemplo, desde el punto de vista terapéutico se ha planteado el uso de "paquetes" específicos orientados a mejorar el manejo de la sepsis $(26,27)$ los cuales, sin embargo, no son todavía de aplicación rutinaria en muchas instituciones. A pesar que los sistemas de puntaje son elementos que han demostrado utilidad diagnóstica en sepsis su uso es poco extendido. La implementación del uso de herramientas basadas en la evidencia es un problema común a nuestras instituciones. Ello involucra no solo el uso de RPC sino incluso el manejo de terapias demostradas como el caso ya mencionado de los protocolos de manejo de sepsis.

Ningún sistema de puntaje discrimina perfectamente el riesgo de muerte o sobrevida en cada paciente sino que permite evaluar el riesgo de muerte o sobrevida en grupos de pacientes y tomar las acciones 0 intervenciones clínicas más apropiadas. Por ejemplo, si un paciente ingresa con shock séptico y es manejado rápidamente en las primeras horas, es probable que sobreviva a pesar de puntajes altos. En la revisión de la literatura no se encontraron estudios nacionales donde se comparen prospectivamente los puntajes MEDS, SOFA, APACHE II y CURB-65, por lo tanto, nuestro estudio es el primero a nivel nacional que compara prospectivamente dichos puntajes en pacientes con sepsis en el servicio de emergencia en una muestra con suficiente poder estadístico para evaluar el uso de los mismos.

Nuestro estudio presenta algunas limitaciones. Hemos tenido una población hospitalaria perteneciente a un hospital de referencia, por lo cual solamente podemos extrapolar las conclusiones a esta población. Es necesario estudios en establecimientos de atención primaria para valorar que tan bueno puede ser el uso de los sistemas de puntaje evaluados en particular el puntaje MEDS debido a su simplicidad. Por otro lado, la alta proporción de pacientes con sepsis grave, shock séptico y fallecidos, podrían limitar la capacidad de extrapolar nuestros resultados a otros establecimientos que atiendan pacientes con cuadros de menor gravedad $\mathrm{y}$, por tanto, ser aplicables fundamentalmente a hospitales de referencia del Ministerio de Salud.

Consideramos que nuestros resultados pueden ayudar a mejorar el manejo de pacientes con sepsis en la emergencia, pues el disponer de RPC puede permitir homogenizar el manejo y generar intervenciones más tempranas en pacientes de alto riesgo. Adicionalmente, la información obtenida a partir de RPC puede permitir informar con sustento objetivo a los familiares sobre el pronóstico del paciente.

En conclusión, los sistemas de puntaje MEDS, SOFA y APACHE II son RPC adecuados para predecir mortalidad y complicaciones en pacientes con diagnóstico de sepsis y deben ser implementados en la atención de emergencias en el Perú. Sin embargo, su utilidad en la práctica dependerá de la capacitación del personal lo cual requiere contar con recursos humanos motivados y preparados para aplicar estas herramientas clínicas. En contraparte, el score CURB-65 tiene un menor rendimiento diagnóstico que los previamente señalados. Por otro lado, se sugiere evaluar el uso de dichos sistemas de puntaje (particularmente el MEDS) en establecimientos de primer nivel a fin de evaluar su utilidad en discriminar aquellos pacientes que deban ser inmediatamente referidos a establecimientos de mayor complejidad.

Contribuciones de autoría: DMM y AST han participado en la concepción y diseño del artículo, análisis e interpretación de datos, redacción y revisión crítica del artículo y aprobación de su versión final. DMM fue responsable del reclutamiento de pacientes y la recolección de datos.

Fuentes de financiamiento: autofinanciado.

Conflictos de interés: los autores declaran no tener conflictos de interés.

\section{REFERENCIAS BIBLIOGRÁFICAS}

1. Crowe C, Kulstad E, Mistry C, Kulstad C. Comparison of severity of illness scoring systems in the prediction of hospital mortality in severe sepsis and septic shock. J Emerg Trauma Shock. 2010;3(4):342-7.

2. Martin GS, Mannino DM, Eaton $S$, Moss M. The epidemiology of sepsis in the United States from
1979 through 2000. N Engl J Med. 2003;348(16):1546-54.

3. Bone RC, Balk RA, Cerra FB, Dellinger $\mathrm{RP}$, Fein AM, Knaus WA, et al. Definitions for sepsis and organ failure and guidelines for the use of innovative therapies in sepsis. The ACCP/SCCM Consensus Conference Committee. American College of Chest Physicians/
Society of Critical Care Medicine. Chest. 1992;101(6):1644-55.

4. Blanco J, Muriel-Bombín A, Sagredo V, Taboada F, Gandía F, Tamayo L, et al. Incidence, organ dysfunction and mortality in severe sepsis: a Spanish multicentre study. Crit Care. 2008;12(6):R158. doi: 10.1186/ cc7157. 
5. Kauss I, Grion C, Cardoso L, Anami E, Nunes L, Ferreira G, et al. The epidemiology of sepsis in a Brazilian teaching hospital. Braz J Infect Dis. 2010;14(3):264-70.

6. Dougnac AL, Mercado MF, Cornejo RR, Cariaga MV, Hernández GP, Andresen $\mathrm{MH}$, et al. Prevalencia de sepsis grave en las Unidades de Cuidado Intensivo. Primer estudio nacional multicéntrico. Rev Méd Chile. 2007;135:620-30.

7. Laupacis A, Sekar N, Stiell IG. Clinical prediction rules. A review and suggested modifications of methodological standards. JAMA. 1997;277(6):488-94.

8. Shapiro NI, Wolfe RE, Moore RB, Smith E, Burdick E, Bates DW. Mortality in Emergency Department Sepsis (MEDS) score: A prospectively derived and validated clinical prediction rule. Crit Care Med. 2003;31(3):670-5.

9. Vincent JL, Moreno R, Takala J, Willatts $\mathrm{S}$, De Mendonça A, Bruining $\mathrm{H}$, et al. The SOFA (Sepsis-related Organ Failure Assessment) score to describe organ dysfunction/failure. On behalf of the Working Group on Sepsis-Related Problems of the European Society of Intensive Care Medicine. Intensive Care Med. 1996;22(7):707-10.

10. Knaus WA, Draper EA, Wagner DP, Zimmerman JE. APACHE II: A severity of disease classification system. Crit Care Med. 1985;13(10):818-29.

11. Lim WS, van der Eerden MM, Laing R, Boersma WG, Karalus N, Town GI, et al. Defining community acquired pneumonia severity on presentation to hospital: an international derivation and validation study. Thorax. 2003;58(5):377-82.

12. Howell MD, Donnino MW, Talmor D, Clardy P, Ngo L, Shapiro NI. Performance of Severity of Illness Scoring Systems in Emergency Department Patients with Infection. Acad Emerg Med. 2007;14(8):709-14.

13. Chen C, Chong C, Liu L, Chen $\mathrm{K}$, Wang $\mathrm{T}$. Risk stratification of severe sepsis patients in the emergency department. Emerg Med J. 2006;23(4):281-5.

14. Ferreira FL, Bota DP, Bross A, Mélot C, Vincent JL. Serial Evaluation of the SOFA score to predict outcome in critically iII patients. JAMA. 2001;286(14):1754-8.

15. Nguyen HB, Banta JE, Cho TW, Van Ginkel C, Burroughs K, Wittlake WA, et al. Mortality predictions using current physiologic scoring systems in patients meeting criteria for early goal-directed therapy and the severe sepsis resuscitation bundle. Shock. 2008;30(1):23-8. doi: 10.1097/ SHK.0b013e3181673826.

16. Jones A, Saak K, Kline J. Performance of the Mortality in emergency department Sepsis score for predicting hospital mortality among patients with severe sepsis and septic shock. Am J Emerg Med. 2008;26(6):689-92.

17. Carpenter CR, Keim SM, Upadhye $S$, Nguyen HB; Best Evidence in Emergency Medicine Investigator Group. Risk stratification of the potentially septic patient in the emergency department: the mortality in the emergency department sepsis (MEDS) score. J Emerg Med. 2009;37(3):319-27. doi: 10.1016/j. jemermed.2009.03.016.

18. Olsson T, Lind L. Comparison of the rapid emergency medicine score and APACHE II in nonsurgical emergency department patients. Acad Emerg Med. 2003;10(10):1040-8.

19. Jones AE, Trzeciak S, Kline JA. The Sequential Organ Failure Assessment score for predicting outcome in patients with severe sepsis and evidence of hypoperfusion at the time of emergency department presentation. Crit Care Med. 2009;37(5):1649-54. doi: 10.1097/CCM.0b013e31819def97.

20. Minne L, Abu-Hanna A, de Jonge E. Evaluation of SOFA-based models for predicting mortality in the ICU: A systematic review. Crit Care. 2008;12(6): R161. doi: 10.1186/cc7160.

21. Sevransky JE, Martin GS, Shanholtz C, Mendez-Tellez PA, Pronovost P, Brower R, et al. Mortality in sepsis versus non-sepsis induced acute lung injury. Crit Care. 2009;13(5): R150. doi: 10.1186/cc8048.

22. Gullo A, Iscra F, DI Capua G, Berlot G, Lucangelo U, Peratoner A, et al. [Systemic and organ dysfunction response during infusion of recombinant human activated protein C (rhAPC) in severe sepsis and septic shock]. Minerva Anestesiol. 2005;71(12):785-801. [Artículo en Inglés e Italiano]

23. Paez Jl, Tengan FM, Barone AA, Levin AS, Costa SF. Factors Associated with mortality in patiens with bloodstream infection and pneumonia due to Stenotrophomonas maltophilia. Eur J Clin Microbiol Infect Dis. 2008;27(10):901-6. doi: 10.1007/ s10096-008-0518-2.
24. Çildir E, Bulut M, Akalin H, Kocabaş E, Ocakoğlu G, Aydin SA. Evaluation of the modified MEDS, MEWS score and Charlson comorbidity index in patients with community acquired sepsis in the emergency department. Intern Emerg Med. 2013;8(3):255-60. doi: 10.1007/s11739-012-0890-x.

25. Nguyen HB, Van Ginkel C, Batech M, Banta J, Corbett SW. Comparison of Predisposition, Insult/Infection, Response, and Organ dysfunction, Acute Physiology And Chronic Health Evaluation II, and Mortality in Emergency Department Sepsis in patients meeting criteria for early goal-directed therapy and the severe sepsis resuscitation bundle. J Crit Care. 2012;27(4):362-9. doi: 10.1016/j. jcrc.2011.08.013.

26. Stoneking L, Denninghoff K, DeLuca L, Keim SM, Munger B. Sepsis bundles and compliance with clinical guidelines. J Intensive Care Med. 2011;26(3):17282. doi: 10.1177/0885066610387988.

27. Romero PC, Hernández PG. Actualización del bundle de reanimación inicial y monitorización integral de la perfusión tisular en la sepsis severa. Rev Med Chil. 2013;141(9):1173-81. doi: 10.4067/ S0034-98872013000900010.

28. Innocenti F, Bianchi S, Guerrini E, Vicidomini S, Conti A, Zanobetti, et al. Prognostic scores for early stratification of septic patients admitted to an emergency departmenthigh dependency unit. Eur J Emerg Med. 2014;21(4):254-9. doi: 10.1097/ MEJ.0000000000000075.

29. Macdonald SP, Arendts G, Fatovich DM, Brown SG. Comparison of PIRO, SOFA, and MEDS scores for predicting mortality in emergency department patients with severe sepsis and septic shock. Acad Emerg Med. 2014;21(11):1257-63. doi: 10.1111/ acem. 12515 .

30. Hilderink MJ, Roest AA, Hermans M, Keulemans YC, Stehouwer CD, Stassen PM. Predictive accuracy and feasibility of risk stratification scores for 28-day mortality of patients with sepsis in an emergency department. Eur J Emerg Med. 2015;22(5):331-7. doi: 10.1097/MEJ.0000000000000185.

\footnotetext{
Correspondencia: Alonso Soto.

Dirección: Departamento de Medicina. Hospital Nacional Hipólito Unanue. Av. Cesar Vallejo 1390. El Agustino, Lima, Perú. Teléfono: (+511) 3627777 Anexo 2151. Correoelectrónico:sotosolari@gmail.com
} 